\title{
7 Wave Functions: Mathematics Is Reality
}

\begin{abstract}
If the ultimate reality of anything is mathematical, it ensues that there must be some kind of 'personal' mathematical 'object', or property, or 'signature', attached to everything and anything, i.e. to any object or person or any definable, correlated collection or assembly of things - in brief, to every conceivable thing, object, or correlated collection of things in the universe. Indeed, that mathematical object must be, in some sense, more real than the object itself, because it must describe and determine that object across the range of all its possible realities, both actualized and potential, within any applicable dimensionality.
\end{abstract}

The term correlated, or entangled, in the context of this book means that the correlated entities share the valid mathematical relationship we touched upon earlier and which we'll discuss further. To reflect real-world reality, such mathematical objects must be able to change and evolve over time. To link up with any new objects to which they may become correlated through physical interactions, they may reversely give up, at least in part, erstwhile associations which have become no longer fully current, and so on.

This mathematical object indeed exists: it is called a wave function (30).

A specific wave function is associated to every particle constituent of matter anywhere and wave functions are also associated with any coherent assembly of particles - that is to say to any collection of particles, atoms, molecules or collections thereof, (aka objects - an atom, two atoms, your brain, your mind, your whole body, you, your neighbour(s), this physical book, this e-Book, a teapot when empty, a teapot when full, the universe itself, etc).

In order to be or become associated within a collective wave function (i.e. a wave function attached to a specific set of constitutive items or elements) there needs to exist some correlation between these elements. For instance, there is no association or correlation whatsoever between, say, a leaf on some tree and a rock on the moon. There is therefore no dedicated wave function linking up these items, even though these two objects would be very loosely correlated within the wave function of the whole universe (also see indented $\$$, note 90). There is, however, a clear association between, say, the atoms within an existing lump of metal, such as a currency coin. These atoms are at the very least correlated by the existence of the electric forces that keep the lump together. They are also spatially correlated - when one atom in the coin is moved in space, all the others concertedly move. There therefore exists a collective wave function grouping up the constituents of that lump of metal - i.e., attached to the lump of metal.

The wave function of something affords an immediate mathematical way to calculate the probability of the presence of that something at any given location in space. Indeed, a somewhat rough-and-ready way to determine whether the separate constituents of some collection are bound up within a valid wave function describing 
jointly that particular collection is whether these constituents are bound up in space: all the parts of a defined ensemble move concomitantly if any one constituent of the set changes position. On the other hand, a plate, say, can be removed off a tabletop without causing the table to move: the plate and the table are hence not part of a simple, unique wave function encompassing both, and only, the table and the plate although they may be constituent parts of a wider, more complex wave function, such as the Universe's (obviously, if the Universe were to disappear, both the table and the plate would disappear too.)

Measurement or observation in quantum mechanics means the establishment of a correlation or entanglement between an observer or measuring apparatus and a measured phenomenon, or more precisely, between the wave function of a measuring apparatus and that of the 'measured' or 'observed' phenomenon. There could be a simple kitchen-top experiment that demonstrates the existence of the wave function attached to your teapot - for instance the teapot that you just filled with water and put on the heating range.

There is an 'old wives' saying' which, interestingly, exists in many different human languages: $a$ watched pot never boils. Of course there is a strong element of the psychological perception of time embedded in this saying - time seems to pass more slowly when one is impatiently looking forward to something pleasurable happening, such as dinner being ready. But this impatience does not quite necessarily account for one hundred percent of the perception of longer time spans - there might be an actual, small, measurable effect; referred to as the Quantum Zeno effect, which can be easily tested by anyone.

The name Quantum Zeno effect was coined (after Zeno of Elea) by the two physicists who first incontrovertibly established that an unstable particle, of which it is known that it decays swiftly, simply never decays if it is observed (or measured) continuously.

At the time it was not obvious that the halting, or 'freezing', of the particle's overdue decay was due to the observers' consciousness watching the particle - it could quite conceivably have been produced by some perturbation of the system caused by, say, the measuring laser used in observing the particle. Yet the effect has since been observed with large systems, without a laser or any other mechanical measuring apparatus being present. You can, albeit somewhat coarsely, test it at home using a pot and a heating range. To do so watch the pot continuously and have someone time yourself from the instant heat is applied to the pot until the moment the water starts to boil. Now perform the same experiment with all parameters being equal - same heat, same pot, same amount of water, etc. but instead go and check briefly, every 25 seconds or so, whether the water has started boiling. Time yourself too.

Here's why it works.

It does not matter whether the measuring apparatus is made up of a laser, or, say, a set of lasers with processing computer, or all of the above including a human observer - say, the operator of the apparatus - as long as human consciousness - the human brain - also has a wave function. In this way the measurement comes from 
the establishment of a relationship between the wave functions of the measuring apparatus and the measured phenomenon. All the myriad other parameters being equal, the water pot's wave function can reflect two states: water boiling, or not boiling. By observing and measuring the pot continuously, you interfere with the pot's wave function to an extent that it makes it less likely that it will become enmeshed - tightly associated - with its environment, in this case the heat underneath it. The immediate effect of your interfering is that the pot becomes less seamlessly able to associate with, or be interfered with, its environment beyond yourself - in technical terms, to cohere with its environment towards the state of 'water boiling'.

The Quantum Zeno effect has also been found to play a part in a number of other astonishing cases, and is suspected in more (31).

Now any wave function, such as the one attached to the water pot in the above experiment, can quickly evolve from its earlier state and thus 'dissipate' away the values of its previous parameters by interacting with its environment - this is what physicists call decoherence. The words 'dissolve and dissipate' are not quite meant in their ordinary sense, rather, they mean here that according to circumstances the wave functions may evolve in such a way that they absorb new influences (or in other words, new variables) from their environment, and they might also shed past associations. By doing so they evolve and fundamentally change.

For instance, if we call some wave function $\Psi$ (psi pronounced sye) which relates to a set, or collection, of two elements which we'll name $a$ and $b$, then we'll write out this wave function, in shorthand, as $\Psi(\mathrm{a}, \mathrm{b})$.

Now let's say that a couple of external agents, which we'll call $x$ and $y$, interfere with and impact $\Psi-\Psi$ decoheres from its formerly exclusive involvement with $x$ and $y$ only and expands its former identity to now become $\Psi(\mathrm{a}, \mathrm{b}, \mathrm{x}, \mathrm{y})$. It could even decohere further to the extent that $a$, say, could become wholly lost to it, and the wave function would then have evolved into $\Psi(\mathrm{b}, \mathrm{x}, \mathrm{y})$, and the element $a$ would have branched out to live its onwards life first as a new wave function altogether, let's call it $\mathrm{Y}(\mathrm{a})$, which could soon become entangled with other and new environmental influences, which we'll call, say, z and zeta, to become Y(a, z, zeta).

There is a key hidden background element in the above, it is the behind-the-scene role played by the deliberate volition of a sentient being in enabling the emergence of the group wave function, here the wave function associated with the water pot. Without somebody filling the teapot with water, the wave function of the system made up by the teapot filled with water would not have come about. Of the fourteen mainstream interpretations of quantum mechanics, two (the Copenhagen interpretation and the Penrose-Ghiradi-Rimini-Weber) attribute a causal role to consciousness, more specifically to the consciousness of any participant involved, and three more interpretations attribute to it a conditional, co-creating or incidental role (32).

Interestingly, if and whenever we try to shy away from a role for consciousness in how reality weaves its own fabric, for instance by favouring an interpretation of 
quantum mechanics that does not give a role to consciousness, such a role somehow seems to always reappear and re-enter the picture, as it were, through some other door. Indeed, although the few interpretations of quantum mechanics which put consciousness at its core and thus call for a sentient consciousness to play its key enabling role, do not seem to constitute its most convincing interpretations, some key role for consciousness seem to become inescapable anywhere we turn. We'll explore this more in detail soon.

But what does the wave function actually materially mean? The short answer is: everything: nothing material has any ultimate materiality but for their associated wave function.

The long answer, explored at length in the literature of physics, is that a given wave function provides a means of calculating everything that pertains to the object this particular wave function represents (even if the actual calculations are as a rule far too complex to perform): importantly, the probability of presence (33) of the object at a given place and time, but also much more besides, including non trivial parameters, such as the likelihood of presence in possible parallel realities, and the likelihood of the existence of such parallel realities to begin with.

Closer to home, the wave function also explains the above-cited mystery of why a football or a bullet would obediently speed off to their intended destinations at the imparted direction and speed and energy without knowledge of such destination or energy. (The foregoing sentence is actually not exactly right: they dash off more or less to their destination at more or less the energy intended - within the error margins of their constant 'recalculating'.)

Here's how: The wave function indirectly indicates the likelihood of presence of its associated object within a certain area of space-time.

The wave function continuously 're-calculates' itself (for lack of a better word), by constantly "calculating” the instant probability of the presence at each and any point of space within the whole universe of the object attached to the wave function in question. The boundary conditions used in the calculation (technically a calculation called an integration) are the geographical limits of the space we are looking for the object in, and within which we calculate the probability of the object's presence. Note that if the size of the universe itself is used to set the boundary conditions of the calculation, then the calculation always yields one, because there is a one in one chance, a one hundred per cent likelihood, that we'll find an object within the universe if that object exists: an existing object has got to be somewhere in the universe (34).

As it evolves through time, the wave function thus 'recalculates' itself at every 'instant' (whatever that means) and takes on an instant, 'spot' value also impacted by the various properties which pertain to the calculation - including the so-called boundary conditions attached to the integral terms. As it happens, these boundary conditions evolve over time - they are newly set at every instant of time, and any drastic change in the object's environment or conditions, such as a kick, immediately 
causes a change in the values of these boundaries. The mathematical effect of the kick was to alter the boundary values governing the integration. The kick did not affect the ball directly, but affected it indirectly, via its associated mathematical reality.

Because of these newly-set boundary values, the self-calculating highest values of the wave function, corresponding to the peak probability of presence of the associated object, position themselves on the desired trajectory. Likewise, all the other associated parameters, such as force, momentum, energy, direction, etc., will manifest, or actualize themselves - show up - into reality with values which statistically will most often lie within very close range of the highest probability values calculated. These parameters are all part of the information embedded within the wave function.

Some information is always lost whenever information is relayed indirectly: it's always better and more accurate to hear someone speak directly than being told by a third party what that person said, or, say, to read an original text rather than a translation.

The same applies here. Experiment have been conducted to observe the trajectories of electrons after they have been emitted. Electrons are so light that any fleeting instantaneous random deviations from an intended trajectory can be observed, and, because the wave functions of electrons can be explicitly calculated, these deviations can be statistically computed and predicted. The random instantaneous deviations that can thus be theoretically predicted have been observed in experiments and they behave exactly as is expected from the calculations - the electrons are observed to continuously stray from their intended path, seeking out their trajectory in real time, and always reverting, more or less, to their intended (imparted) trajectory. The statistics of the experimentally observed deviations from the imparted trajectories, in terms of the directions and amplitudes of deviations, match within a very narrow margin the values expected from the calculations of the wave functions (35).

A question of course is what is it that prompts the wave function's self-recalculation to launch, stop, and begin again? Is there a mechanism that allows this to happen, to in effect isolate and privilege a given succession of otherwise indistinguishable timepoints when a wave function executes a continuing series of recalculations? If time is discrete, it is relatively easy to conceptualize that a recalculation is reset at each actual instant of present time. If time is continuous, what mechanism triggers the 'recalculation' and when?

In any space-time, where for whatever reason, the recalculation mechanism would not be enabled, reality would become weird and objects (if they could exist in such a space-time) would not be able to self-calculate their own wave functions - they would drift about aimlessly, regardless of any past history. A kicked ball or a fired bullet would just float about rudderlessly. Matter as we know it would be unable to exist in such a universe in the first place: such a universe would only ever be able to host virtual matter. 
Regardless of whether time is continuous or not, there does exist a zone where time simply stops: the spherical two-dimensional 'event horizon' around a black hole (also called the Schwarzschild horizon, after astronomer Karl Schwarzschild.) Any two-dimensional object, perfectly precisely poised at a Schwarzschild horizon would be unable to recalculate its wave function (quantum effects however would soon destroy the perfect poise there.) We'll revisit black holes shortly (36).

Since consciousness keeps putting in appearances, be it at the core of certain interpretations or at their outer edges, it is time we briefly looked at the material seat of human consciousness: the brain, which indisputably is... 